\title{
RESPONSABILIDAD SOCIAL UNIVERSITARIA, ÉTICA EMPRESARIAL Y SU APLICACIÓN EN LOS PROCESOS DE FORMACIÓN EN LAS INSTITUCIONES DE EDUCACIÓN SUPERIOR
}

\author{
UNIVERSITY SOCIAL RESPONSIBILITY, BUSINESS ETHICS AND ITS APPLICATION IN IN THE \\ TRAINING PROCESSES IN HIGHER EDUCATION INSTITUTIONS
}

\author{
Carlos Mauricio De La Torre-Lazcano ${ }^{1}$ (i) \\ ${ }^{1}$ Universidad Central del Ecuador, Ecuador.Email:cdelatorre@uce.edu.ec
}

Para citar este artículo: De la Torre, L. C. (2019). Responsabilidad Social Universitaria, ética empresarial y su aplicación en los procesos de formación en las Instituciones de Educación Superior. Clío América, 13(26), 370-379. doi: http://dx.doi.org/ $\underline{10.21676 / 23897848.3611}$

Recibido: 27 mayo de 2019

Aceptado: 16 de septiembre de 2019

Publicado en línea: noviembre 28 de 2019

\section{Palabras clave: deshonestida \\ d; ética profesional; formación profesional; responsabili dad; universidad.}

JEL: M14; I23

\section{RESUMEN}

Esta investigación se desarrolla con la finalidad de determinar la problemática social relacionada con la falta de valores éticos y morales a nivel académico. Al ser un estudio cualitativo, recurre a la revisión bibliográfica documental como principal fuente para la obtención de información, sustentando técnicamente que la implementación en los procesos de formación profesional que aborden la ética empresarial como elemento trascendental puede minimizar los actos de corrupción. El estudio tiene tres referentes: responsabilidad social universitaria, ética empresarial y su aplicación en los procesos de formación en las instituciones de educación superior. El análisis determina que en la responsabilidad social existe un recurrente cometimiento de actividades delictivas con la participación de funcionarios públicos y privados, que las universidades desarrollan una formación profesional centrada en habilidades técnicas, con poco contenido ético y moral. De ahí emerge la necesidad de fortalecer el proceso de instauración ético y moral en la formación universitaria, incorporando unidades de análisis relacionadas con la deontología o abordando esta disciplina como eje transversal en todas las asignaturas del currículo universitario. Por tanto, se concluye que existe una directa relación entre responsabilidad social universitaria, ética empresarial y la necesidad de su aplicación en los procesos de formación profesional.

\section{ABSTRACT}

This research is carried out with the aim of determining the social problem related to the lack of ethical and moral values at the academic level. Being a qualitative study, it uses the documentary bibliographic review as the main source for obtaining information, technically sustaining that the implementation in professional training processes that address business ethics as a transcendental element, can minimize corruption actions. The study has three references: university social responsibility, business ethics and its application in training processes in higher education institutions. The analysis determines that in social responsibility there is a recurring commitment of criminal activities with the participation of public and private officials, that universities develop professional training focused on technical skills, with little ethical and moral content. Hence, the need emerges to strengthen the ethical and moral establishment process in university education, incorporating units of analysis related to deontology or approaching this discipline as a transversal axis in all the subjects of the university curriculum. Therefore, it is concluded that there is a direct relationship between university social responsibility, business ethics and the need for its application in professional training processes. 


\section{RESPONSABILIDAD SOCIAL UNIVERSITARIA, ÉTICA EMPRESARIAL Y SU APLICACIÓN EN LOS PROCESOS DE FORMACIÓN EN LAS INSTITUCIONES DE EDUCACIÓN SUPERIOR}

\section{INTRODUCCIÓN}

"Nos movemos en un tiempo globalizado, marcado por un cambio en la visión de la ética y la conciencia moral" (Saltos, 1999, p. 23). Los constantes escándalos en Iberoamérica debidos a actos deshonestos dados a conocer por los denominados Panamá Papers, la investigación de corrupción denominada Lava Jato, Odebrecht, entre otros, han responsabilizado a altos ejecutivos de compañías, muchos líderes políticos y mandatarios latinoamericanos han terminado en prisión y otros se encuentran prófugos de la justicia. Estos hechos, sin duda han conmocionado a toda la sociedad. Las actividades ilícitas perpetradas utilizando diversos artilugios se siguen conociendo y ya se empiezan a sentir sus devastadores efectos. La corrupción se engendra en la supremacía del poder del Estado en todos sus niveles, incluyendo el sector privado (Bautista, 2014). Las actuaciones carentes de ética y moral de las personas y particularmente de ciertas autoridades públicas y políticos, están empañadas de corrupción y ello se percibe en todos los estratos de la sociedad.

Mora (2013) señala que cada vez se siente más la exigencia del compromiso que debe existir para enfrentar problemas actuales como la pobreza a causa de la distribución inequitativa de la riqueza, abuso de poder, deficiente moral pública de políticos e inadecuada conducta de los empresarios; en general, aspectos negativos que afectan la institucionalidad de los Estados. Se asume que la ética "como convicción interna o actitud, un compromiso personal de vivir con arreglo a unos valores vinculantes, unas normas definidas y unos principios personales fundamentales o virtudes" (Küng, 2011, p. 41). Es necesario que, las personas se cobijen de sus principios en todo momento y espacio, y más aún cuando de servir a la sociedad se trate.

La ética no es un valor agregado, es un valor intrínseco de todas las actividades humanas, entre las cuales están la económica, la académica y la empresarial. Téngase en cuenta que, cualquier acción a nivel personal o social está mediada por la condición humana, por lo que se ratifica la conciencia de invocar un comportamiento enmarcado en la moral. "La ética, como dijimos antes, no presenta una descripción exterior de la conducta humana, sino que propone un ideal. Y el ideal es algo más, bastante más que una idea o que una articulación de ideas" (Savater, 1995, p. 49). Las organizaciones desarrollan sus actividades para las que fueron creadas dentro de un marco normativo nacional e institucional, pero existen estándares internacionales emitidos y diseñados por organismos supranacionales que pueden o deben ser adoptados en virtud de las buenas prácticas. Arbogast (2017) determina que los empresarios se enfrentan a complejas situaciones cotidianas donde se pueden presentar dilemas éticos sin contar con la suficiente experiencia o capacitación. Lo cual constituye un importante punto de referencia, que guía un accionar responsable en lo personal y social, el desarrollo de las personas y de la sociedad debe estar cimentado en conductas de responsabilidad y corresponsabilidad.

Es común observar una limitada aplicación de valores éticos y morales en los distintos ámbitos de la sociedad, entre los cuales sobresale la educación, a pesar de que "la escuela y los maestros, como reproductores de los valores de la sociedad, deben ser el paradigma en su actividad y ejercicio cotidiano de los valores de la ética" (González, 2005 , p. 167). En el ámbito académico, el estudio de la responsabilidad social corporativa (RSC) ha ganado espacio debido a la urgente necesidad de transparencia, debido a la omisión de directrices concretas de ética empresarial, en el momento en que las escuelas de administración determinan como obligatorio implementar dentro de sus planes de estudios cursos sobre responsabilidad social empresarial (RSE) y cuando más empresas se disputan la adopción de políticas responsables. Por ello, en la actualidad la credibilidad en dichas organizaciones y la legalidad de la RSE parecerían tocar fondo, ya que la falsedad sería lo que predomina en ese ambiente (Montoya y Martínez, 2012).

Podría decirse que las instituciones de educación superior (IES) han descuidado la dimensión ética y moral de los estudiantes en los procesos de formación profesional, en prácticamente todas las áreas del conocimiento, ya que han centrado sus esfuerzos académicos en proporcionar o robustecer aspectos teóricos, operativos y técnicos, lo que incrementa notablemente la preocupación sobre el papel de la ética en la educación universitaria (García y Cerón, 2005). La academia de manera específica, en virtud de su naturaleza que radica en la generación de conocimientos, destrezas, competencias y valores de los actuales profesionales y del futuro, deberían "ser las primeras en esforzarse por ser socialmente responsables y sustentables. Además, que ello se lleve a cabo nítidamente y de manera evidente a la luz pública" (Rebeil, 2015, p. 19). Se hace énfasis en los procesos de transparencia de la información y acceso a la misma que la ciudadanía debería tener para vigilar los modos de proceder de los funcionarios públicos en la toma de decisiones y la ejecución de acciones de interés social.

En este contexto global donde de manera persistente la carencia de ética y moral campea en las decisiones políticas 
y el ejercicio profesional, el rol de la academia no puede ser indiferente, por lo que aparece la denominada responsabilidad social universitaria (RSU) debido a que la universidad se constituye en el espacio de formación humanística y profesional de más alta jerarquía. Acotando que el proceso de enseñanza - aprendizaje no solamente recae en las IES, sino que inicia desde la formación temprana del ser humano en cuyo proceso está involucrada toda la sociedad. Vallaeys (2007) señala que la RSU:

es una nueva filosofía de gestión universitaria que pretende renovar el compromiso social de la Universidad al mismo tiempo que facilitar soluciones innovadoras a los retos que enfrenta la educación superior en el contexto de un mundo globalizado pero insostenible en sus patrones de desarrollo (p. 2).

Lo anterior ratifica la necesidad de realizar estudios serios en torno a las relaciones existentes entre responsabilidad social universitaria, la ética empresarial y su aplicación en los procesos de formación de las IES, como mecanismos para una apropiada gestión en los ámbitos de su competencia.

\section{METODOLOGÍA}

Esta investigación es de enfoque cualitativo, ya que para demostrar la trascendencia que tiene la aplicación de la ética en el desarrollo de actividades profesionales y la interrelación existente entre la RSC y los procesos de formación en las universidades se recurrió a un amplio análisis documental que llevó a la reflexión de las diversas relaciones e interrelaciones de las variables. Para Hernández, Fernández y Baptista (2014), "la investigación cualitativa se enfoca a comprender y profundizar los fenómenos, explorándolos desde la perspectiva de los participantes en un ambiente natural y en relación con el contexto" (p. 364). Por tanto, se orienta hacia la comprensión de la realidad estudiada, cuyos resultados al ser difundidos ampliamente aportarán en el desarrollo de la conciencia social con respecto al manejo de la cosa pública.

La trascendencia del tema y su implicación radica en las actividades académicas, que contemplan la formación profesional en los diversos programas de pregrado y posgrado, en torno a la obligatoriedad de desarrollar y fortalecer la formación de valores, debido a la falta de ética existente en los ámbitos tanto público como privado. Si bien se pueden ubicar muchas fuentes bibliográficas que versan sobre RSE, este estudio se ha centrado principalmente en las que toman como protagonistas a las IES, en la denominada RSU en torno a la implementación de sus planes de estudio y la incidencia en la formación de los futuros profesionales.

\section{RESULTADOS}

\section{Necesidad de aplicación ética en la actividad profesional}

Resulta lógico suponer que todas las actividades profesionales que desarrolla el ser humano de manera general deberían ser realizadas en el marco de la honestidad. Por lo que existe desde el punto de vista ético un especial interés en que la práctica sea cumplida bajo esos requerimientos de orden natural, "como es evidente, la praxis se desarrolla bajo coordenadas espaciotemporales definidas. La ética atiende a la obligación moral en su validez universal, mientras que son las situaciones particulares las que reclaman una reflexión moral" (García y González, 2014, p. 150). Entendiendo que la ética en profundidad genera una transformación en la conducta de quien adquiere ese conocimiento. Bautista (2015) alude a que dentro de ese proceso de transformación las personas atraviesan por las siguientes fases: "a) reflexiona y delibera, b) adquiere conciencia, c) distingue entre lo conveniente y nocivo, d) adopta principios sanos, e) asume deberes de manera voluntaria y no por obligación, f) logra madurez de juicio" (p. 59). En tanto esta conciencia ética se va asimilando, se logra una renovación en el comportamiento de los profesionales, estas modificaciones graduales de orden axiomático los motiva a actuar de forma íntegra en el cumplimiento de sus actividades. Luego del análisis documental, se determina que la formación a nivel universitario resultaría carente de ética y moral; aunque se haya prestado importante atención a la capacitación técnico profesional, no es menos cierto que actualmente el currículo universitario contiene menos formación ética y moral que en tiempos pasados.

Para Bolívar (2005) "la ética profesional comprende el conjunto de principios morales y modos de actuar éticos en un ámbito profesional, forma parte de lo que se puede llamar ética aplicada" (p. 96), en tanto todas las profesiones están inmersas en diferentes áreas del conocimiento con aplicaciones específicas, propias de cada carrera que se desarrollan en diferentes ámbitos, lugares y cada una de ellas requiere ciertas competencias pero todas requieren conductas éticas globales. Por otra parte, y de manera complementaria, la ética de la empresa (EE) puede definirse como "la disciplina que discierne lo que es bueno, recomendable, correcto en la administración de las empresas capitalistas, desde un punto de vista ético y moral. Por su naturaleza ella debe ser una herramienta de la administración de la empresa" (Cuevas, 2009, p. 329). Elementos complementarios que deben formar parte de un 


\section{RESPONSABILIDAD SOCIAL UNIVERSITARIA, ÉTICA EMPRESARIAL Y SU APLICACIÓN EN LOS PROCESOS DE FORMACIÓN EN LAS INSTITUCIONES DE EDUCACIÓN SUPERIOR}

escenario global que propicie actitudes honestas en ambientes profesionales desarrollados bajo la óptica de RSE. Teniendo en cuenta que en el currículo universitario de formación profesional no se cuenta de manera suficiente con contenidos relacionados con la ética y la moral, y al analizar la existencia de innumerables casos de corrupción en el manejo de la empresa pública y privada se deduce que la deficiente formación ética y moral en los procesos educativos universitarios está directamente relacionada con el cometimiento de actos de corrupción en el contexto investigado.

La ética y la moral constituyen aspectos esenciales de la formación integral del ser humano, mucho más si se toma en cuenta la importancia de la obligatoriedad de ejercer actividades profesionales en el marco de los valores. Una cultura ética robusta y la integridad, determinan un umbral moral para los funcionarios y gerentes previniendo que infrinjan la ley, lo que reduce el comportamiento ilegal (Huisman, 2019). La deontología desde un punto de vista legal es definida por Carretero (2010) como "la ciencia o rama autónoma que estudia los deberes profesionales de un colectivo, público o no, que regulan su comportamiento, debidamente aprobados por los órganos pertinentes para ello, y que, de no cumplirse, implican sanciones" (p. 2), mismas que deben ser revisadas de manera constante por las autoridades correspondientes. El ámbito de la deontología consiste "en la diferencia entre las intenciones y las consecuencias de los actos. En esta medida, no se es responsable por las consecuencias que resulten de haber actuado en favor de una norma moral, inclusive si son indeseadas" (Uribe, 2002, p. 286). Dentro del ámbito empresarial, Soto y Cárdenas (2007) determinan que "uno de los factores más importantes para el éxito o fracaso de un directivo es su habilidad para el análisis de problemas y la toma de decisiones" (p. 20). Por lo expuesto y tomando en consideración el estudio bibliográfico realizado, surge la necesidad de desarrollar una formación profesional con importante contenido deontológico, que, si bien no necesariamente hará constar la asignatura de deontología en el currículo de todos los niveles educativos de formación profesional, no es menos cierto que como está, debe constituir un eje transversal abordado en prácticamente todas las asignaturas del currículo universitario.

\section{RSC y la adopción imprescindible de ética en la educación superior}

En la medida en que la organización logre ser económica, legal y moralmente responsable, y logre también justificar este enfoque para todos los agentes internos, tendrá acceso a un capital que es tan importante como la confianza y la reputación (Calvo, 2013). Con la finalidad de crear un diálogo permanente y constructivo, muchas organizaciones han creado comités de ética, Torralba y Rosàs (2017) consideran que "la ética aplicada solo tiene sentido si está anidada dentro de un diálogo abierto y honesto entre profesionales de diferentes disciplinas que desean un orden más justo" (p. 217).

Para que estos objetivos (totalmente realizables) dejen de ser meras expectativas y puedan conseguirse, se requieren políticas públicas de fomento de la cultura de la RSE en sentido amplio, tomando en consideración los factores que inciden en la simbiosis que debe estar presente entre las empresas, la sociedad y la academia desde la formación inicial. "La formación en los niveles educativos básicos en el marco de una educación en valores de la ciudadanía. La formación universitaria y la formación posgrado de nuestros universitarios y de nuestros técnicos en gestión empresarial" (Jáuregui, 2010, p. 25). La libertad académica para explorar cuestiones importantes y controvertidas es una condición previa esencial para cumplir con la misión de la academia de educar a los estudiantes y avanzar en el conocimiento (Association of American Colleges and Universities, 2006). País (2015) acota que "una universidad que falla en preparar líderes y los profesionales del mañana no es socialmente responsable, no importa en cuántos buenos trabajos esté involucrada" (pp. 195-196).

Las IES tienen la enorme responsabilidad de generar y difundir conocimiento, todo ello dentro de una prolija y compleja articulación social, toda vez que deben fomentar de manera permanente la formación de profesionales competentes. Seres humanos capacitados con valores éticos y morales que respondan a las expectativas y demandas que la colectividad requiere, tornándose entonces necesario conocer si el trabajo ejecutado por la academia cubre las expectativas de la comunidad (Díaz y García, 2011). La interacción de las IES dentro de la sociedad en su conjunto se plasma en una múltiple relación intrínseca, que abarca, por un lado, estudios de orden técnico, y por otro, diferentes conocimientos (inclusive de orden cultural propio) para resolver los nuevos desafíos que se presentan en múltiples y variados conglomerados (sociales, políticos, empresariales, étnicos, etc.) con los cuales la academia se interrelaciona de manera constante (Cantú, 2013).

Dentro de este contexto, la relación de políticas empresariales de la RSC con la cooperación de desarrollo en el ámbito de la educación y formación, tiene inmerso el "desarrollo de habilidades profesionales (directivas, técnicas, etc.) en el personal local, desarrollo de profesionales, potenciación y retención del capital humano 
nacional (evitando la fuga de cerebros), prevención de accidentes laborales, alfabetización de comunidades locales, empoderamiento social" (Navarro y García, 2009, p. 127). La educación superior es un factor esencial para el desarrollo de las sociedades. Los estudiantes acuden a las IES "buscando formación profesionalizante, que les proporcione medios para lograr puestos de trabajo y capacitación para la vida con reconocimiento social" (Carpintero, 2014, p. 62). Por lo que se les debe ofrecer, entonces, formación especializada que cumpla las expectativas del mercado laboral y la sociedad, aspectos sin duda relevantes, pero esencialmente enmarcados en valores morales basados en el ejemplo y la ética.

En tal sentido y teniendo en cuenta que la universidad como institución creada por la sociedad con la finalidad de formar profesionales para esta, resulta indispensable que exista una relación directa entre la universidad y la empresa. La práctica de valores fortalecida en los procesos de formación universitarios y concretada en el accionar de las personas en su vida laboral, adquirirá significado y sentido puesto que el abordaje de diferentes situaciones y problemas profesionales estará caracterizado por la práctica de valores éticos y morales, en cuyo caso la deontología como eje transversal de la formación profesional constituye el origen de buenas prácticas profesionales en el ámbito público y privado.

\section{Interrelación existente entre RSC y RSU}

Schvarstein (2003) señala que una organización es socialmente responsable cuando ha logrado establecer un cúmulo de praxis de orden tanto voluntario como obligatorio, con la finalidad de que sus integrantes y en general la sociedad, hayan cubierto sus obligaciones sociales, incluyendo en ese grupo aquellas necesidades básicas, como educación, salud, seguridad, vivienda o acceso a la justicia. Importante acotación, mas se debe recordar que la finalidad de las empresas para su creación radica en la obtención de rentabilidad, lucro o beneficio para sus accionistas. Lo óptimo radica en que exista en las organizaciones empresariales esa visión social de responsabilidad y que se mantenga en la ejecución de sus operaciones a lo largo del tiempo.

Por otro lado, específicamente en el ámbito académico, "la RSU se entiende como un conjunto de valores y principios que han de orientar las políticas y acciones de la universidad, la cual debe adoptar, desarrollar y transmitir valores y principios éticos a la sociedad" (Martí et al., 2015, p. 71). Para este fin, tiene que canalizarse adecuadamente la imperativa necesidad de cumplir a cabalidad sus obligaciones para con la ciudadanía, robusteciendo de esta manera la relación inmanente entre la academia y la comunidad. Por ello, se puede deducir claramente que no sea posible que exista la RSU sin la RSC y viceversa. No obstante, De la Cruz y Sasia (2008) determinan que

la universidad necesita un discurso propio sobre responsabilidad, que no solo esté en coherencia con los ejes básicos de la misión universitaria, sino que sirva además de marco de referencia para poder comprender cuál es el compromiso de la universidad por la responsabilidad y de qué manera está respondiendo a los retos que hoy la responsabilidad le presenta a la universidad en el marco de la sociedad que la acoge (p. 22).

La RSU también se puede entender como la capacidad que poseen las IES de "aplicar un conjunto de principios y valores en la realización de sus funciones básicas: (1) formación académica y pedagógica, (2) investigación y difusión, (3) gestión de la organización y (4) participación social" (González et al., 2016, p. 8), tomando en consideración que la demanda de los diferentes intervinientes de la sociedad debe ser canalizada de manera adecuada, mediante la aplicación de esquemas claros y transparentes. Deben distinguirse tres niveles en la RSU: “a) RSU interna, que incorpora a los estudiantes, docentes, investigadores, directivos y personal administrativo; b) RSU externa, incluye empleadores, egresados, proveedores y socios estratégicos directos; c) RSU extra, hacia el Estado, la sociedad, el desarrollo y el medio ambiente global" (Gasca y Olvera, 2011, p. 50).

En virtud del análisis precedente, se puede determinar que la RSU tiene un ámbito de aplicación holístico y se "plantean diferentes aspectos que se van acercando hacia un concepto integrador que recoge todos esos elementos: compromiso social, valores, desarrollo sustentable, formación de profesionales competentes y ciudadanos responsables, etc." (Gaete, 2012, p. 133). Martí y Vargas (2014) señalan que la relación entre educación superior y el "ámbito empresarial no puede soslayarse, mucho menos en pleno auge de la globalización, la dinámica de integración de la educación superior a nivel internacional es un proceso para garantizar la competitividad de las regiones" (p. 55). De hecho, el rol de las IES "no debe ser pasivo ante los continuos hechos plagados de deshonestidad, el fortalecimiento de la necesidad de la ética aplicada al mundo de las profesiones se torna en un verdadero desafío y una exigencia imperativa" (De la Torre, 2017, p. 12). Martí et al. (2015) determinan "la importancia de incorporar investigación sobre RSU en docentes e investigadores, puesto que los valores y principios que transmiten son los que forman a la futura 
generación de profesionales" (p. 78).

La realidad social y natural debe ser examinada globalmente. Es decir, teniendo en cuenta que los elementos que las conforman se encuentran relacionados de manera dialéctica, compleja y holística. De ahí que los hechos que se presentan a nivel social aun cuando aparentemente son aislados, son consecuencia de aquello que se hace, o de aquello que deja de hacerse. Desde otra perspectiva, si en la universidad se desarrolla una limitada formación ética y moral, habrá de esperarse que en la sociedad se presenten actos de inhibición, negligencia, inobservancia y corrupción. Por tanto, si ello está sucediendo en los actuales momentos históricos, es fundamental que la sociedad regrese su mirada a la universidad y en su sentido más amplio, a la escuela, no para responsabilizar, sino para promover los cambios y transformaciones que se requieren en la causa, es decir, la universidad, la academia como encargada de la formación de los ciudadanos.

\section{DISCUSIÓN}

De los resultados se deduce que el currículo universitario evidencia una limitada formación ética y moral de los estudiantes, lo cual estaría relacionado con la presencia constante de actos de corrupción en la administración y la gestión de la empresa pública y privada. Con el estudio se constata que los profesionales actúan en un contrasentido, inclusive al margen de la ley, por lo que se torna imperativa la aplicación ética en el ejercicio profesional. "Las acusaciones de irresponsabilidad de líderes empresariales como factor crítico en las crisis han creado una intensa demanda social por una revisión de la educación gerencial que está recorriendo el mundo" (Kliksberg, 2013, p. 167). La incidencia de profesionales en actos de corrupción por acción u omisión en todos los sectores es alarmante y contribuye al progreso de la delincuencia organizada.

Cortina (2009) determina que "la sociedad civil se sitúa más allá del Estado y del mercado, en la esfera de la opinión pública, atravesada por las redes del lenguaje que generan solidaridad" (p. 115). Las teorías éticas asumen el hecho de que las relaciones entre la empresa y la sociedad se fundamentan en valores éticos y que las empresas deben aceptar las responsabilidades sociales, por sobre cualquier otro tipo de consideración, como una obligación moral. Desde esta perspectiva, sería la que se está apostando efectivamente por un auténtico desarrollo sostenible, no solo estratégico sino también moral y normativo (Fernández, 2014). La confianza en la academia debe ser recuperada, su finalidad debe seguir cumpliéndose con una renovación integral de sus estructuras y el cumplimiento cabal de sus actividades, esas valiosas tareas "que ha dejado de hacer o simplemente las que nunca ha podido realizar a plenitud, pero sobre todo es necesario restablecer el vínculo entre la educación superior en América Latina con el desarrollo social y humano" (Gasca y Olvera, 2011, p. 42).

Este estudio implica la reformulación del quehacer universitario en función de los intereses superiores. De ahí que la revisión de las acciones y omisiones en torno a los procesos de formación profesional en las IES constituiría un mecanismo para reconocer fortalezas, oportunidades, debilidades y amenazas tanto de la universidad como de la sociedad en su conjunto. Es decir, de acuerdo con las necesidades, intereses y motivaciones de la sociedad. Al desarrollar estas acciones de manera seria y responsable, existe la posibilidad de que la sociedad recupere la confianza en la universidad. Por el contrario, se seguirá manteniendo la idea de que la formación profesional está limitada al desarrollo de habilidades, destrezas e inclusive competencias técnico profesionales, mas no aporta lo suficiente para una formación humana y basada en la práctica de valores.

Dentro de este análisis no se debe perder de vista que los valores que dinamizan las conductas adecuadas de todos los estamentos universitarios intervinientes, deben estar plasmados en un documento que debe ser prolijamente sistematizado y socializado como uno de los pilares fundamentales en la generación de confianza en las IES. En la concepción de estos nuevos códigos de ética deben integrarse esquemas muy bien fundamentados, en los que la ética y la RSU establezcan que el sistema de cumplimiento deje de ser una cuestión estrictamente jurídica, para convertirse en un proceso de participación (García-Marzá, 2017). "El código ético o de conducta debe recoger una serie de principios y valores que han de ser asumidos por toda la comunidad universitaria" (González, Fontaneda y Camino, 2017, p. 29). Si bien las universidades cuentan con organismos y comisiones encargadas de la ética y la moral en la gestión universitaria, no es menos cierto que estas deben concretar acciones de socialización. De tal manera que las normas, acuerdos, regulaciones y más posibilidades que determinan los modos o maneras de actuar en el contexto universitario, más allá de que consten en los diferentes instrumentos legales, impregnen la conciencia de los actores universitarios y garanticen la adecuada concreción de relaciones e interrelaciones entre estos. 


\section{CONCLUSION}

Si bien se ha podido establecer que existe una relación intrínseca entre la RSC y la RSU, se debe señalar que existe mucho trabajo por realizar, especialmente para empoderar a la ciudadanía como actor principal en la determinación de la pertinencia de los programas académicos que las universidades ofertan, toda vez que existe un cúmulo de necesidades de la colectividad que deben ser solventadas por las IES, generando la obligatoriedad de contar con cantidades superiores de investigadores para cubrir las cada vez mayores exigencias de la sociedad, especialmente en Latinoamérica debido a la existente desigualdad en la distribución de la riqueza.

La ética mantiene su vigencia y universalidad, ya que resulta inverosímil conocer la magnitud del impacto económico que alcanzan los actos plagados de deshonestidad política, ideológica, económica, técnica, entre otras muchas. Los problemas de corrupción son generalizados por todo el mundo y cada vez aumentan más, perjudicando lamentablemente a las clases sociales más vulnerables. Se debe proveer a los estudiantes universitarios (futuros profesionales en proceso de formación), lineamientos claros sobre el complejo escenario global actual, la afectación en el ejercicio profesional presente y futuro por la falta de ética, mediante la aplicación de un modelo transversal en todas las áreas del conocimiento y en todas las actividades universitarias (incluyendo rendición de cuentas permanente por parte de estas, la denominada accountability), tareas que aún se encuentran en espera de ejecución efectiva por parte de las IES, para propender una reconstrucción global de su esencia. Por lo que una de las acciones de responsabilidad de las universidades y de manera específica en los programas de negocios o empresariales, resulta la implementación de procesos de formación en donde los estudiantes aborden la ética empresarial como elemento trascendental en el proceso de enseñanza - aprendizaje, de tal manera que se puedan minimizar los actos de corrupción que están presentes tanto en el sector público como privado. Este nuevo enfoque debe ser innovador, revolucionario y adoptar medidas tecnológicas, involucrando a todos los estamentos universitarios, sociedad y empresa, ya que resulta totalmente lógico que las organizaciones busquen trascender a lo largo del tiempo; justamente un claro ejemplo de esta permanencia y legado son las universidades, mismas que han venido cumpliendo parcialmente sus objetivos, y es que como se ha manifestado en este análisis, existen muchas actividades pendientes de ejecución que debemos seguir esforzándonos para que se efectúen.

\section{Declaración sobre conflictos de interés}

El autor de la presente investigación declara que las afirmaciones realizadas, las ideas planteadas, así como las opiniones, discusiones y conclusiones emitidas son responsabilidad exclusiva del autor de la misma y son independientes de su institución de filiación. De igual manera, durante el desarrollo de la investigación y la redacción de este trabajo no se ha incurrido en ningún conflicto de interés.

\section{REFERENCIAS BIBLIOGRÁFICAS}

Arbogast, S. (2017). Resisting Corporate Corruption: Cases in Practical Ethics from Enron Through the Financial Crisis. Wiley. https://doi.org/10.1002/9781119323679

Association of American Colleges and Universities. (2006). Academic Freedom and Educational Responsibility. AACU. https://www.aacu.org/sites/default/files/files/ab out/academicFreedom.pdf

Bautista, O. (2015). Máximas clásicas para interiorizar la ética. Centro de Investigación en Ciencias Sociales y Humanidades, Universidad Autónoma del Estado de México. http://ri.uaemex.mx/handle/20.500.11799/5822 $\underline{4}$

Bautista, D. (2014). Globalización y corrupción. Previniendo la corrupción en Iberoamérica mediante la ética pública en los albores del siglo XXI. Dilemata, 15, 118-129. http://www.dilemata.net/revista/index.php/dile mata/article/viewFile/295/315

Bolívar, A. (2005). El lugar de la ética profesional en la formación universitaria. Revista Mexicana de Investigación Educativa, 10(24), 93-123.

http://www.scielo.org.mx/scielo.php?script=sci _arttext\&pid=S1405-

$66662005000100093 \& \operatorname{lng}=\mathrm{es} \&$ tlng=es

Calvo, P. (2013). CSR in the internal management of organizations. Ramon Llull Journal of Applied Ethics, 4, 87-104. http://www.raco.cat/index.php/rljae/article/view 
RESPONSABILIDAD SOCIAL UNIVERSITARIA, ÉTICA EMPRESARIAL Y SU APLICACIÓN EN LOS PROCESOS DE FORMACIÓN EN LAS INSTITUCIONES DE EDUCACIÓN SUPERIOR

\section{/281038/368694}

Cantú, P. (2013). Las instituciones educación superior y la responsabilidad social en el marco de la sustentabilidad. Revista Electrónica Educare, $\quad$ 17(3), 41-55. http://www.revistas.una.ac.cr/index.php/EDUC ARE/article/view/5239

Carpintero, H. (2014). Hacia una universidad sostenible y socialmente responsable. En A. Cortina (Coord.), La responsabilidad ética de sociedad civil (págs. 59-79). Cajamar Caja Rural.

http://www.publicacionescajamar.es/pdf/public aciones-periodicas/mediterraneoeconomico/26/mediterraneo-economico-26.pdf

Carretero, S. (2010). Corrupción, funcionarios públicos y papel de la deontología. Revista Telemática de Filosofía del Derecho, 13, 1-26. http://www.rtfd.es/numero13/01-13.pdf

Cortina, A. (2009). Ética de la Empresa: No sólo Responsabilidad Social. Revista Portuguesa de Filosofia, 65 , 113-127. http://www.jstor.org/stable/41220792

Cuevas, R. (2009). Ética y responsabilidad social de la empresa: su concepción del hombre. Polis, 8(23), 323-349. https://scielo.conicyt.cl/pdf/polis/v8n23/art15.p $\underline{\text { df }}$

De la Cruz, C. y Sasia, P. (2008). La responsabilidad de la universidad en el proyecto de construcción de una sociedad. Revista Educación Superior y Sociedad, 13(2), 17-52. http://ess.iesalc.unesco.org.ve/ess3/index.php/e ss/article/view/39

De La Torre, C. (2017). Convenios internacionales sobre corrupción y la necesidad ética de cumplimiento global. XXII Congreso Internacional de Contaduría, Administración e Informática.

http://congreso.investiga.fca.unam.mx/docs/xxii /docs/11.01.pdf
Díaz, I. y García, M. (2011). Más allá del paradigma de la alfabetización. La adquisición de cultura científica como reto educativo. Formación Universitaria, 4(2), 3-14. https://doi.org/10.4067/S0718$\underline{50062011000200002}$

Fernández, J. (2014). Por una conexión de la RSE con la ética empresarial. Revista Icade, 91, 145-171.

https://doi.org/10.14422/icade.i91.y2014.006

Gaete, R. (2012). Responsabilidad social universitaria: una nueva mirada a la relación de la universidad con la sociedad desde la perspectiva de las partes interesadas. Un estudio de caso (Tesis doctoral, departamento Sociología y Trabajo Social, Universidad de Valladolid, https://uvadoc.uva.es/handle/10324/923

García-Marzá, D. (2017). Responsabilidad Social Universitaria un sistema de ética y cumplimiento para la universidad. En A. Andrés, S. Barberá y D. Pallarés (Eds.), Nuevas perspectivas en la gestión de la responsabilidad social en las universidades. Publicacions Universitat Jaume I. http://hdl.handle.net/10234/170505

García-Marzá, D. y González, E. (2014). Ética. Publicacions Universitat Jaume I. http://hdl.handle.net/10234/80726

García, C. y Cerón, A. (2005). Entre la ética y deontología profesionales. Reflexión sobre el campo periodístico. Reencuentro, 43, 44-51. http://www.redalyc.org/articulo.oa? id=3400430 $\underline{6}$

Gasca, E. y Olvera, J. (2011). Construir ciudadanía desde las universidades, responsabilidad social universitaria y desafíos ante el siglo XXI. Convergencia, 56, 37-58. https://convergencia.uaemex.mx/article/view/11 $\underline{19}$ 
González, O., Fontaneda, I. y Camino, M. (2017). La responsabilidad social universitaria: retos y oportunidades. En A. Andrés, S. Barberá y D. Pallarés (Eds.), Nuevas perspectivas en la gestión de la responsabilidad social en las universidades. Publicacions Universitat Jaume I. http://hdl.handle.net/10234/170505

González, O., Fontaneda, I., Camino, M. y Revilla, A. (2016). Responsabilidad Social en las Universidades: del conocimiento a la acción. FORÉTICA.

http://www.foretica.org/informe_ejecutivo_rsu. pdf

González, E. (2005). La corrupción: patología colectiva. Instituto Nacional de Administración Pública.

Hernández, R., Fernández, C., y Baptista, M. (2014). Metodología de la investigación. McGrawHill.

Huisman, W. (2019). Blurred Lines: Collusions Between Legitimate and Illegitimate Organizations. En The Handbook of WhiteCollar Crime (págs. 139-158). Wiley. https://doi.org/10.1002/9781118775004.ch10

Jáuregui, R. (2010). Reputación corporativa, RSC, sostenibilidad, acción social... ¿de qué hablamos cuando hablamos de esto? En M. Arráez y P. Francés (Eds.), Ética y responsabilidad ante la crisis, (págs. 19-32). Ediciones Sider.

Kliksberg, B. (2013). Ética para empresarios. Ética y Economía.

Küng, H. (2011). La crisis económica global hace necesaria una ética global. El Manifiesto por una ética económica global. En BBVA (Ed.), Valores y ética para el siglo XXI (págs. 35-57). BBVA.

https://www.bbvaopenmind.com/libro/valoresy-etica-para-el-siglo-xxi/

Martí, J., Mendes, A. y Campos, P. (2015). Los principios de la responsabilidad social universitaria en la UNED (España). Sinapsis, 7(7),

70-79.

http://www.eam.edu.co/ojs/index.php/sinapsis/a rticle/view/78

Martí, J. y Vargas O. (2014). La responsabilidad social de las universidades y su inserción en el contexto iberoamericano. Relevancia de la Carta de Río - 2014. Cuadernos de RSO, 3(2), 53-62. https://ucu.edu.uy/sites/default/files/facultad/fce /rso/RSO_vol3_Marti_y_Vargas.pdf

Montoya, B. y Martínez P. (2012). Responsabilidad Social Empresarial: Una respuesta ética ante los desafíos globales. Fundación Konrad Adenauer. http://www.kas.de/wf/doc/kas_37565-1522-430.pdf?140425034037

Mora, J. (2013). Ética aplicada al mundo de las profesiones. Una propuesta académica para universitarios del siglo XXI. Centro de Publicaciones PUCE.

Navarro, F. y García-Marzá, D. (2009). La RSC en el marco de la cooperación al desarrollo y la internacionalización de la empresa española en países de renta media y rehabilitación postbélica. Revista Española del Tercer Sector, 11, 115143.

http://www.plataformatercersector.es/sites/defa ult/files/N11\%20RETS\%20Las\%20ONG\%20d e\%20Desarrollo.pdf

País, E. (2015). Humanismo Empresarial, ética $y$ responsabilidad social en el pensamiento de Peter Drucker (Tesis doctoral, departament d'Economia i Organització d'Empreses). Universitat Internacional de Catalunya, España. http://www.tdx.cat/handle/10803/384009

Rebeil, M. (2015). Responsabilidad social organizacional, imperativo ético para las instituciones de educación superior. Evolución, 38 ,

17-20.

https://www.dropbox.com/s/j3hbx2ns7v8ni4f/E voluci\%C3\%B3n\%20IARSE\%20N\%C2\%BA3 8\%20- 
RESPONSABILIDAD SOCIAL UNIVERSITARIA, ÉTICA EMPRESARIAL Y SU APLICACIÓN EN LOS PROCESOS DE FORMACIÓN EN LAS INSTITUCIONES DE EDUCACIÓN SUPERIOR

\%20Edici\%C3\%B3n\%20Octubre\%202015.pdf ?dl=0

Saltos, N. (1999). Ética y corrupción. Estudio de casos. Respondanet.

Savater, F. (1995). Invitación a la ética. Anagrama.

Schvarstein, L. (2003). La inteligencia social de las organizaciones. Paidós.

Soto, E. y Cárdenas, J. (2007). Ética en las organizaciones. McGrawHill.

Torralba, F. y Rosàs, M. (2017). Ethical deliberation within organizations. Ramon Llull Journal of Applied Ethics, 8, 207-223. http://www.raco.cat/index.php/rljae/article/view /321879/412509

Uribe, A. (2002). El punto de vista moral en las empresas: una descripción del uso deontológico de la razón. Signos Filosóficos, 8, 277-290. http://signosfilosoficos.izt.uam.mx/index.php/S $\underline{\text { F/article/view/759 }}$

Vallaeys, F. (2007). Responsabilidad Social Universitaria. Propuesta para una definición madura y eficiente. Programa para la Formación en Humanidades, 1-11. Tecnológico de

http://www.bibliotecavirtual.info/wpcontent/uploads/2011/12/Responsabilidad_Soci al_Universitaria_Francois_Vallaeys.pdf 